\title{
Frontal Lobe Anaplastic Oligodendroglioma
}

National Cancer Institute

\section{Source}

National Cancer Institute. Frontal Lobe Anaplastic Oligodendroglioma. NCI Thesaurus.

Code C156120.

An anaplastic oligodendroglioma occurring in the frontal lobe. 